\title{
Background Oriented Schlieren Applied to Study Shock Spacing in a Screeching Circular Jet
}

\author{
Michelle M. Clem ${ }^{1}$, Khairul B.M.Q. Zaman ${ }^{2}$, Amy F. Fagan ${ }^{3}$ \\ NASA Glenn Research Center, Cleveland, Ohio, 44135
}

Background oriented schlieren (BOS) is a recent development of the schlieren and shadowgraph methods. The BOS technique has the ability to provide visualizations of the density gradient in both the axial and radial directions. The resultant magnitude of the density gradients allows for comparison with shadowgraph images. This paper first compares data obtained by the BOS and shadowgraph techniques at identical conditions in a free jet. The patterns and spacing of the shock trains obtained by the two techniques are found to be consistent with one another. This provides confidence in the shock spacing measurement by the BOS technique. Due to its simpler setup, BOS is then applied to investigate the shock spacing associated with the screech phenomenon, especially during stage jumps. Screech frequencies from a $37.6 \mathbf{~ m m}$ convergent nozzle, as a function of jet Mach number $\left(M_{j}\right)$, are shown to exhibit various stages. As many as eight stages are identified with the present nozzle over the range $1.0<M_{j}<1.7$. BOS images are acquired at various screech conditions and the shock spacing is examined as a function of $M_{j}$.

$\begin{array}{ll} & \\ \varepsilon & =\text { angle through which light is refracted } \\ S & =\text { refractive index gradient } \\ \Delta x & =\text { apparent pixel shift due to refraction } \\ M_{j} & =\text { jet Mach number } \\ M_{D} & =\text { design Mach number }\end{array}$

I. Introduction

The aim of the present investigation is first to assess the effectiveness of measuring shock spacing using the BOS technique by comparing its results with data from the conventional shadowgraph technique and then applying it to study shock spacing associated with the screech phenomenon. The BOS technique and aspects of the screech phenomenon are reviewed in the following sections.

\section{A. Background Oriented Schlieren}

Background oriented schlieren (BOS) is a recent development of the schlieren and shadowgraph techniques, which are used to non-intrusively visualize density gradients. Schlieren and shadowgraph methods provide qualitative information of the first and second derivatives of the density in the flow, respectively. However, schlieren and shadowgraph techniques can be difficult, time consuming and costly, as they require large mirrors or lenses and precise alignment. Conversely, BOS captures the density field but only requires a CCD camera, light source, and a background, making it a much simpler and less costly option for flow visualization ${ }^{1}$.

BOS is based on background distortion or an apparent movement of the background when imaged through a density field onto a detector plane ${ }^{2}$. Figure 1 illustrates the principle of BOS. An incoherent light source uniformly illuminates a background composed of a high-contrast random pattern that is imaged by a camera and lens system. Without any refractive index gradient (denoted ' $\mathrm{S}$ ' in Fig. 1) in the optical path, light will travel from the background undisturbed. The dashed green line, along the optical axis in Fig. 1, illustrates an individual light ray traveling from a point on the background to the camera undisturbed in the case without a refractive index gradient present. When acquiring an image under this condition, the background will look undistorted. A refractive index gradient in the path causes light to refract. The solid green line in Fig. 1 shows the light ray being refracted by the gradient at 'S'. Now when the background is imaged through the non-uniform density field, points on the

\footnotetext{
${ }^{1}$ Research Engineer, Optical Instrumentation and NDE Branch, 21000 Brookpark Road

${ }^{2}$ Research Engineer, Inlet and Nozzle Branch, 21000 Brookpark Road, AIAA Associate Fellow

${ }^{3}$ Research Engineer, Optical Instrumentation and NDE Branch, 21000 Brookpark Road, AIAA Senior Member
} 
background will appear shifted by ' $\Delta \mathrm{x}$ ' at the detector plane. Therefore, when acquiring BOS data it is necessary to take two images: a reference image prior to the refractive index gradient being present and an image after the light has refracted through the density field. For maximum sensitivity the refractive index gradient should occur halfway between the camera and the background ${ }^{2}$.

The shift between the two acquired images caused by the refracted light can be calculated by correlation methods. Existing algorithms that have already been developed for particle image velocimetry (PIV) are used to determine the background image displacements within small sub-regions across the image ${ }^{3}$. Unique to BOS, the density gradient in two orthogonal directions in the plane of the background can be distinguished from one another.

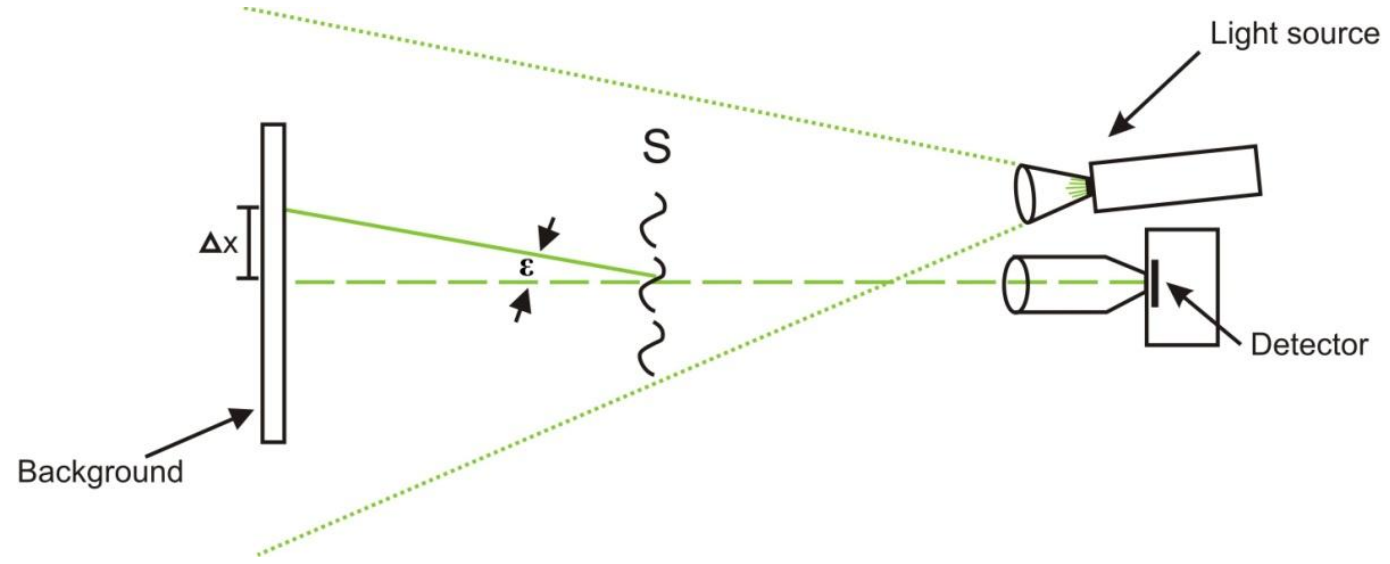

Fig. 1 Illustration of the principle of BOS, showing light passing through a refractive index gradient (S) resulting in a displacement of $\Delta x$. This displacement appears as a pixel shift in the acquired images.

\section{B. Screech}

Supersonic jets operating at imperfectly expanded conditions generate noise, which is comprised of three basic components: turbulent mixing noise, broadband shock-associated noise and screech tones. ${ }^{4}$ The latter two noise components are associated with the quasi-periodic shock cell structures formed in the jet plume. The screech phenomenon, involving discrete frequency tones, is the primary focus of the BOS application in this study.

Alan Powell first reported detailed observations of screech tones and offered an explanation for their occurrence based on acoustic feedback phenomenon ${ }^{5}$. The feedback mechanism responsible for screech tone generation has continued to be studied for several decades ${ }^{6,7}$. The feedback loop begins in the thin jet mixing layer, near the nozzle lip, where it is most receptive to external excitation. Acoustic disturbances traveling upstream impinge on this area and excite the inherent instability waves. While propagating downstream the instability wave grows and when sufficiently amplified it interacts with one of the quasi-periodic shock cells, generating a disturbance. The disturbance travels outside of the jet back upstream, impinges on the jet mixing layer near the nozzle lip and excites new instability waves, thus closing the feedback loop. The process is accompanied by the generation of an intense tone that is referred to as screech.

As the screech tone is emitted, the jet flow undergoes strong oscillations. The oscillation can be in toroidal mode or in helical/flapping mode. The flapping mode oscillations consist of equal amounts of left and right helical mode oscillations. It has been known that the dominant screech tone goes through mode-switching or stage-jumps as the Jet Mach number $\left(M_{j}\right)$ is increased. The frequency within a given stage decreases continuously as $M_{j}$ is increased. In previous studies with circular nozzles ${ }^{8,9}$ flow field oscillations in the axisymmetric (stages $A_{1}$ and $A_{2}$ ), flapping (stages B and D), and helical (stage C) modes have been observed. However, the B and D stages may be unstable, switching between the flapping and helical oscillations. The staging behavior will become clearer with the frequency data presented in the following. However, it is fair to say that the screech mechanism has remained from being completely understood due to its unsteady, intermittent nature and its dependence on a variety of parameters. One of the unknowns is the behavior of the shock spacing during a stage jump. It is not clear if the shock spacing adjusts to accommodate the new wavelength associated with a new stage or if it remains unchanged while other parameters adjust to accommodate the new stage. In this paper, we examine the shock spacing variations using BOS. 


\section{Experimental Setup and Procedure}

The experiments were conducted in a large open jet facility at NASA Glenn Research Center, shown in Fig. 2(a). Compressed air is supplied through one end of the $760 \mathrm{~mm}$ diameter plenum chamber fitted with flow conditioning units. The air discharges through the nozzle on the other end of the plenum into the quiescent ambient of the test chamber. All data in this paper pertain to unheated flow. Figure 2(b) shows a $50.8 \mathrm{~mm}$ diameter convergentdivergent nozzle with a design Mach number $\left(M_{D}\right)$ of 1.8 which was previously used to acquire shadowgraph data in connection with internal shock study. ${ }^{10}$ BOS data obtained with this nozzle is first compared to the shadowgraph data. Figure 2(c) shows a $37.6 \mathrm{~mm}$ diameter convergent nozzle used for the subsequent screech study.

a.)

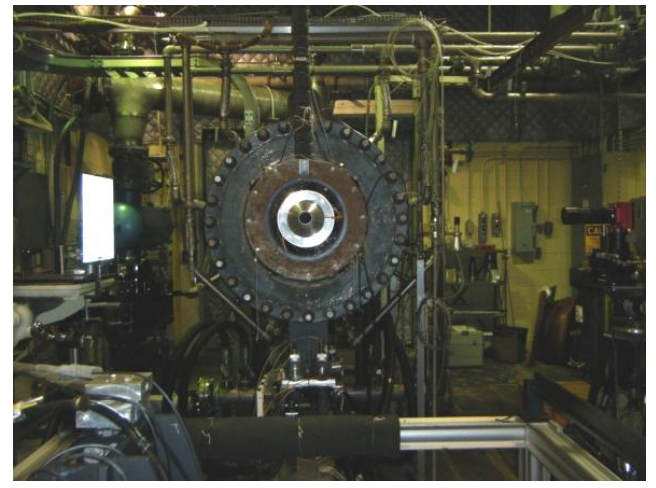

b.)

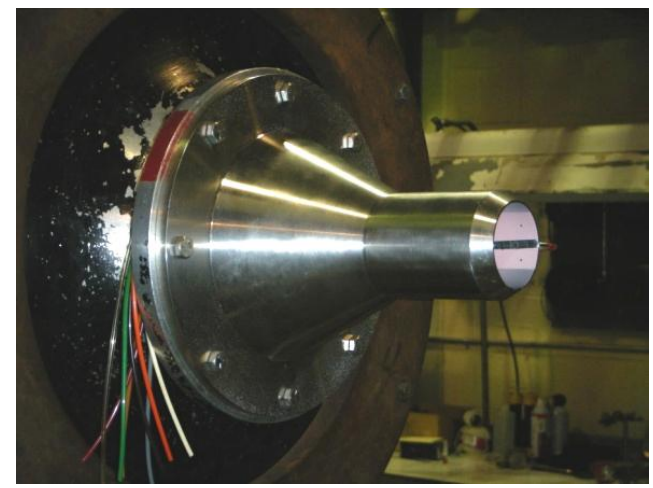

c.)

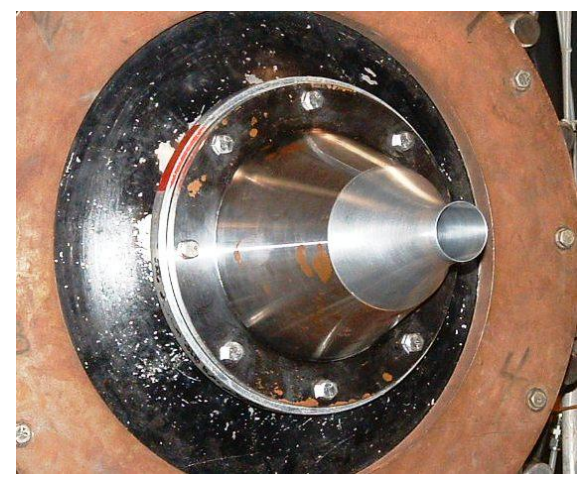

Fig. 2 Experimental facility. (a) Open jet rig, (b) $50.8 \mathrm{~mm}, M_{D}=1.8$, convergent-divergent nozzle used to compare BOS and shadowgraph data, (c) $37.6 \mathrm{~mm}$ convergent nozzle used to study screech phenomenon.

A top-view diagram of the BOS setup with relevant dimensions is shown in Fig. 3. A $10 \mathrm{~W}, 530 \mathrm{~nm}$ light emitting diode (LED) is used as the incident light source for the system. Light from the LED is uniformly spread across the $0.3 \mathrm{~m} \times 0.3 \mathrm{~m}$ background, consisting of a random speckle pattern of retro-reflective paint particles, which directs the incident light back onto itself and towards the camera. The retro-reflective paint consists of thousands of tiny glass beads bonded to the painted surface, creating a high-contrast background. The image of the illuminated background is focused onto the camera detector by a Nikon $200 \mathrm{~mm}$ lens. The scientific-grade CCD camera is a Princeton Instruments model EC 11000 which has a detector with 4008 x 2672 pixels and a pixel size of $9 \mu \mathrm{m} \times 9 \mu \mathrm{m}$. The 12-bit images were captured with a $30 \mathrm{~ms}$ exposure time. The large detector size enabled capture of the desired field of view within the distances dictated by the constraints of the test facility. The background was placed at a distance of $1.74 \mathrm{~m}$ from the focusing lens of the camera. The distance from the camera lens to the nozzle was $0.984 \mathrm{~m}$ and the distance from the background to the nozzle was $0.756 \mathrm{~m}$.

To obtain the BOS data, image pairs were acquired over a range of $M_{j}$. A reference image was acquired prior to the flow being turned on and the data image was acquired upon reaching the desired condition. The pixel shifts, in both the axial $(x)$ and radial $(y)$ directions, between the two images were calculated using PIV software. Using Tecplot, the pixel shifts were plotted as contours, which enabled visualization of the density gradients in the axial and radial directions. The magnitude of the resultant density gradients was plotted and compared with the shadowgraph data taken at the same conditions. 


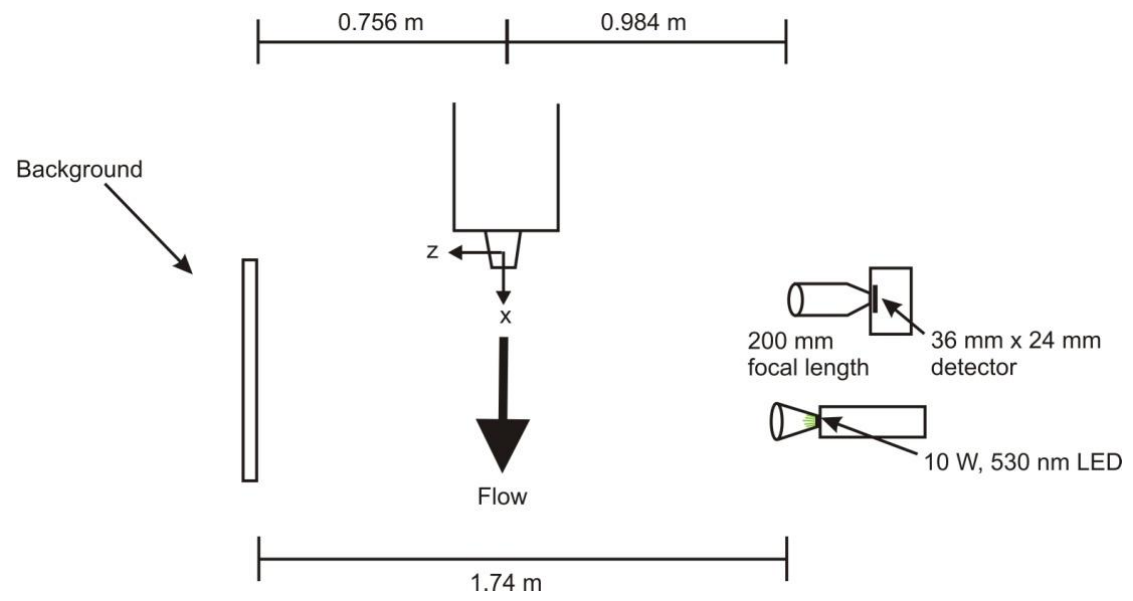

Fig. 3 Top view schematic of the BOS setup.

\section{Results}

\section{A. BOS Data Compared to Previously Acquired Shadowgraph Data}

As stated before, the BOS data images were acquired with the $50.8 \mathrm{~mm}$ diameter, $M_{D}=1.8$ nozzle (designated as 'M18L' in Ref. 10). The images were obtained over the range $1.2<M_{j}<1.7$ at matching test conditions of the previous shadowgraph experiment reported in the cited reference. An example of the BOS data at $M_{j}=1.6$ is shown in Fig. 4. The pixel shifts measured in the $x$-direction, corresponding to the axial density gradient, is shown in Fig. 4(a). Pixel shifts in the y-direction, corresponding to the radial density gradient, are shown in Fig. 4(b). These figures illustrate the usefulness of BOS for obtaining individual components of the density field in a single snapshot. The magnitude of the two components is shown in Fig. 4(c); this is the quantity that was used for comparison with the shadowgraph data.

Examples of shadowgraph images at $M_{j}=1.2,1.4$, and 1.6 are displayed in Figs. 5(a)-5(c), respectively. Corresponding BOS images, taken for the same values of $M_{j}$ are displayed in Figs. 6(a)-6(c), respectively. It can be seen that the flow and shock boundaries are sharper in the shadowgraph data than in the BOS data. Also the BOS images contain some asymmetry. It is believed that with further refinement of the BOS technique, the image quality and asymmetry can be improved upon. Presently however, the overall agreement of the images obtained by the two techniques is good, particularly the inferred shock spacings as described further in the following paragraphs.

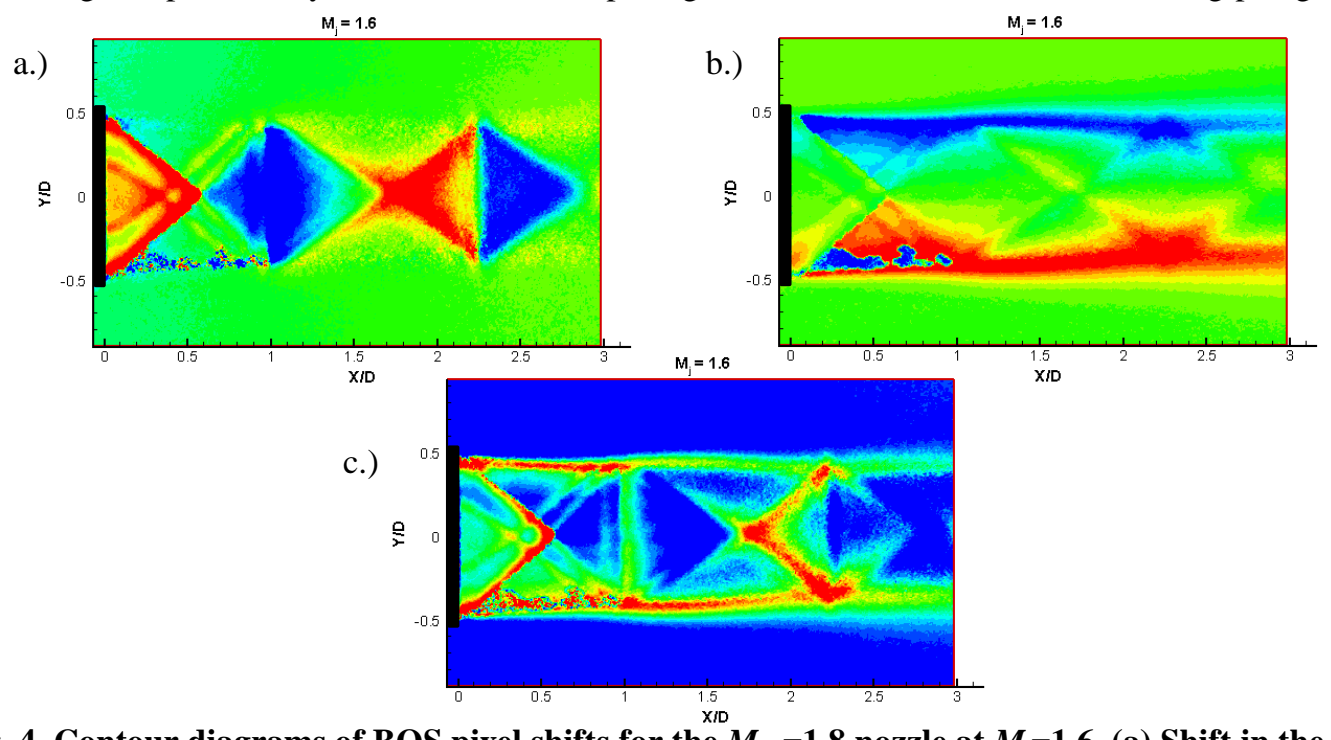

Fig. 4 Contour diagrams of BOS pixel shifts for the $M_{D}=1.8$ nozzle at $M_{j}=1.6$. (a) Shift in the axial (x) direction, (b) in radial $(y)$ direction and (c) the resultant magnitude. 


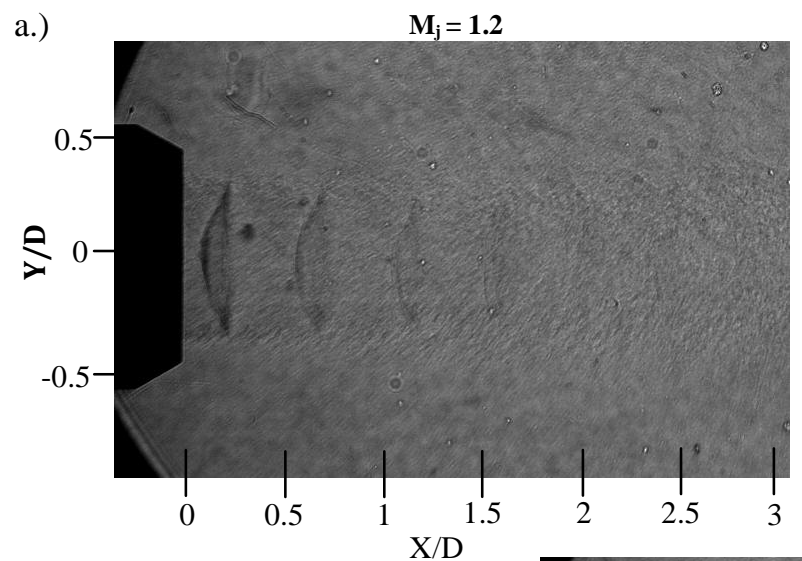

b.)

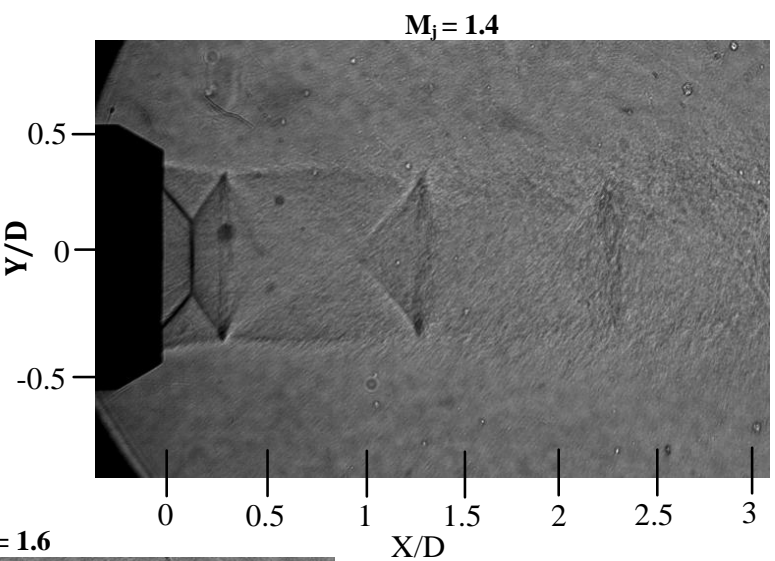

c.)

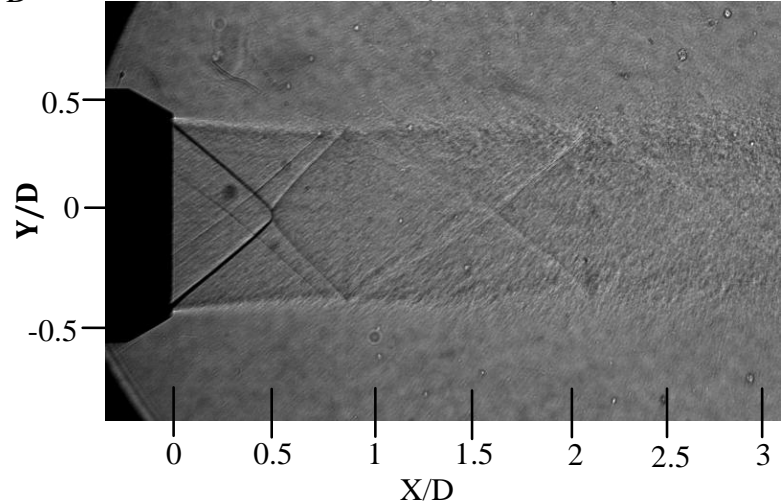

Fig. 5 Shadowgraph images for the $M_{D}=1.8$ nozzle at (a) $M_{j}=1.2$, (b) $M_{j}=1.4$ and (c) $M_{j}=1.6$ acquired previously. ${ }^{10}$

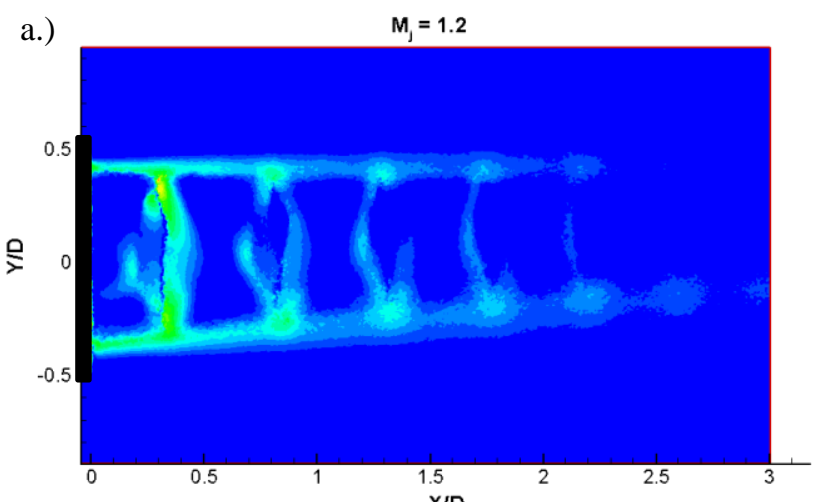

b.) $\quad M_{\mathrm{J}}=1.4$

c.)

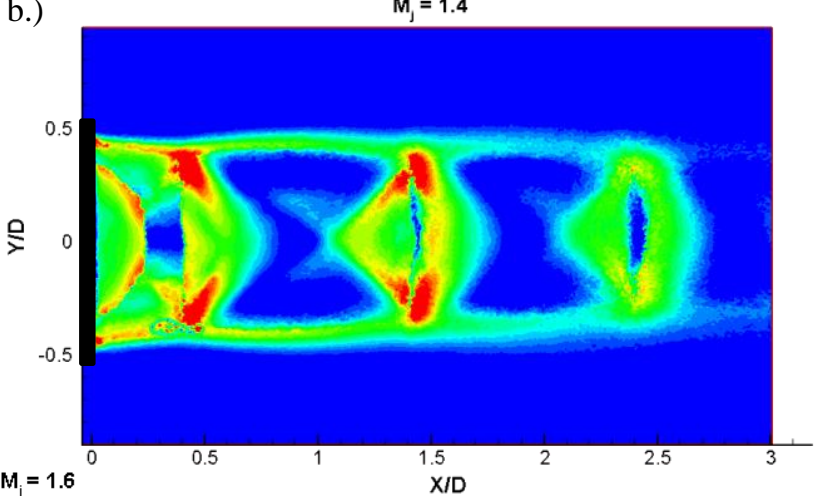

c.)

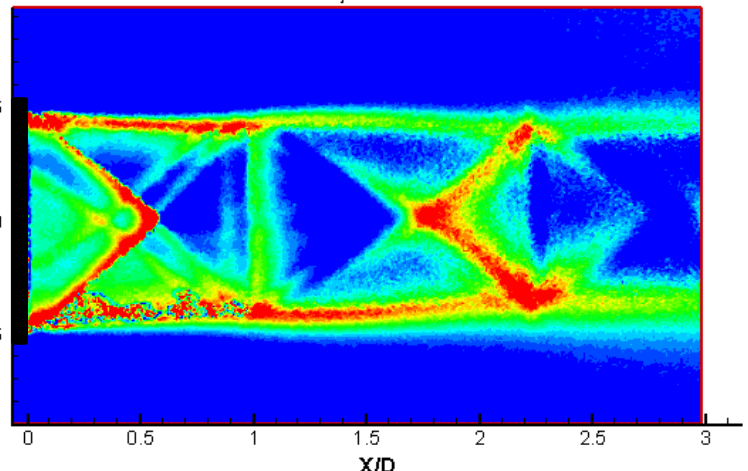

Fig. 6 BOS images for the $M_{D}=1.8$ nozzle at (a) $M_{j}=1.2$, (b) $M_{j}=1.4$ and (c) $M_{j}=1.6$. 
The shock spacing is examined in Fig. 7 for $M_{j}=1.4$. The tips of the shock at the jet's shear layer are marked by arrows. Average of the first two tip-to-tip spacings is taken to be the shock spacing. This is deemed justified because the spacing is not expected to change much within the first two cells, while an average provides a better representative value. As it can be seen from the labels in the figure, the average spacing obtained by the two techniques is in good agreement.

It is important to note the subjectiveness in the determination of the shock tip location with either the BOS or the shadowgraph technique. The uncertainty in the shock spacing measurement with the BOS technique in Fig. 7 is estimated to be $7.6 \%$ whereas the uncertainty in the shadowgraph shock spacing is estimated to be $3.5 \%$. The shadowgraph results in images that contain data at every pixel resulting in a spatial resolution of $0.248 \mathrm{~mm} / \mathrm{pixel}$, resulting in lower measurement uncertainty. Background oriented schlieren produces images that have an averaged data value for every 8 pixels by 8 pixels sub-region, resulting in a spatial resolution of $0.365 \mathrm{~mm} / \mathrm{subregion}$. Note that the camera used in the BOS experiment had much larger sensor with 10.5 times more pixels than the camera used in the shadowgraph experiment. Although, the spatial resolutions between the two techniques are comparable, the difference in the images can be attributed to the different setups, light sources, field of views, dynamic ranges and sensitivities to the density gradients present in these shock-laden flows.

Figure 8 shows the comparison of shock spacing versus $M_{j}$ over the range $1.2<M_{j}<1.7$ obtained by the two techniques for the $M_{D}=1.8$ nozzle. Shock spacing calculated from the shadowgraph and BOS images are consistent within the data uncertainty and follow the expected trend previously observed in Ref. 6 . As $M_{j}$ increases the shock spacing increases as well. These results provide confidence in the shock spacing measurement by the BOS technique. The results on the study of shock spacing in a screeching jet are discussed in the next section.

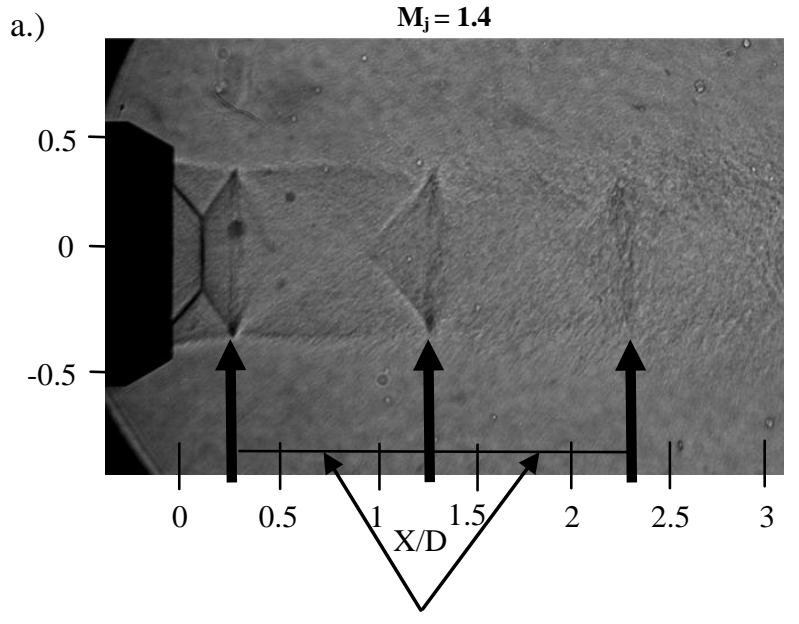

Average shock spacing $=0.965 D$ b.)

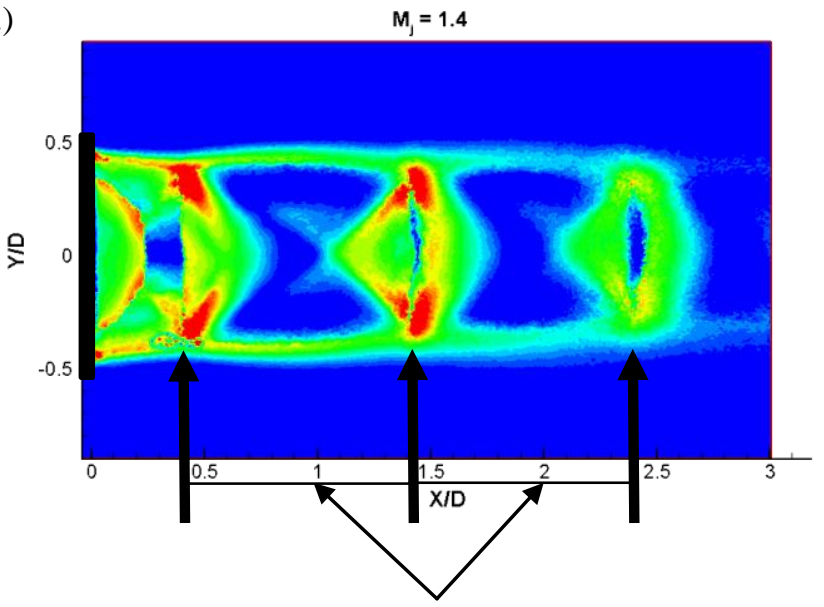

Average shock spacing $=0.945 D$

Fig. 7 Average shock spacing determined from (a) shadowgraph and (b) BOS for $M_{D}=1.8$ nozzle at $M_{j}=1.4$.

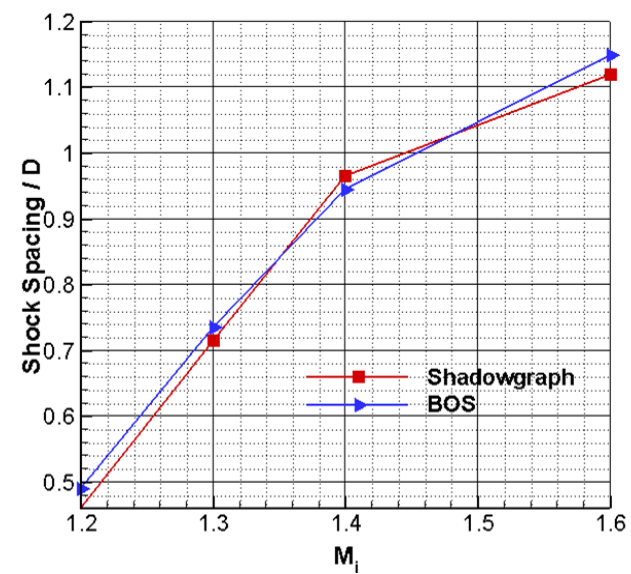

Fig. 8 Non-dimensional shock spacing calculated from shadowgraph and BOS images as a function of $M_{j}$ for the $M_{D}=1.8$ nozzle. 


\section{B. Screech Data}

The screech phenomenon was studied with a $37.6 \mathrm{~mm}$ diameter circular convergent nozzle. The convergent nozzle was chosen since it pertains to most of the previous studies in the literature on the subject. In order to investigate the role of shock spacing in mode-switching or stage jumps, data were obtained with special attention to regions of stage jumps involving hysteresis. First, screech frequency data were acquired by spectrum analysis of the signal from a microphone positioned at $25^{\circ}$ relative to the jet axis. Figure 9 shows the recorded screech frequencies plotted versus $M_{j}$ depicting the various screech stages that were observed. Stages $\mathrm{A}_{1}$ and $\mathrm{A}_{2}$ (axisymmetric), B and D (flapping), and C (helical) have been previously noted in the literature. Stages B and D have also been observed to be unstable and switch between the flapping and helical modes. The B stage (flapping) overlaps with C stage (helical) and the D stage (flapping) overlaps with $\mathrm{C}$ stage. In each of these overlap regions there is hysteresis, i.e., the stage jump occurs at a different location depending on whether $M_{j}$ is increased or decreased. Thus, within a jump region, data could be obtained at exactly the same $M_{j}$ but in different stages. Note that Fig. 9 also shows additional screech frequency stages not previously observed, to the best of the authors' knowledge. These are labeled as B', E, and F. Within the $M_{j}$-range covered, previous studies noted only up to stage D. It appears the screech stages are dependent on details of the nozzle and the reason for this discrepancy remains unclear at this time.

As stated before, when two screech stages overlap there is usually hysteresis. As $M_{j}$ is increased the screech tone frequency in a particular stage decreases and jumps to a new stage at a particular $M_{j}$; however, as $M_{j}$ is subsequently decreased the return to the earlier stage takes place at a lower $M_{j}$. Examples of sound pressure level spectra are shown in Fig. 10; (the same color codes are used for the spectral traces as for the stages in Fig.9). Figure 10(a) shows spectra for stages $A_{1}$ and $A_{2}$. For this stage jump hysteresis was not clear. On the other hand, Figs. 10(b)-(f) show spectra pairs in regions involving clear hysteresis for jumps in stages $\left(B^{\prime}, B\right),(B, C),(C, D),(D, E)$, and $(E$, F), respectively. Of note is Fig. 10(c) where stages B and C are captured at exactly the same frequency depending on increasing or decreasing $M_{j}$.

It was theorized that the shock spacing would display an abrupt change at different stages of the $M_{j}$ values where the hysteresis occurred. In order to investigate this, BOS data were acquired for conditions corresponding to the different data points of Fig. 9 and representative images are displayed in Figs. 11(a) - 11(1). Figure 11(a) shows an image without any perceptible screech tones present. Figures 11(b) - 11(f) show images for screech stages, $A_{1}, A_{2}$, $\mathrm{B}^{\prime}, \mathrm{B}$ ' $+\mathrm{B}$, and $\mathrm{B}$, respectively. The rest of the images in Fig. 11 show pairs in the overlapping (hysteresis) regions where the adjacent stages could be captured at the same $M_{j}$. Thus, Figs. 11(g)-11(l) represent image pairs in the hysteresis regions for stages (C, D), (D, E), and (E, F), respectively.

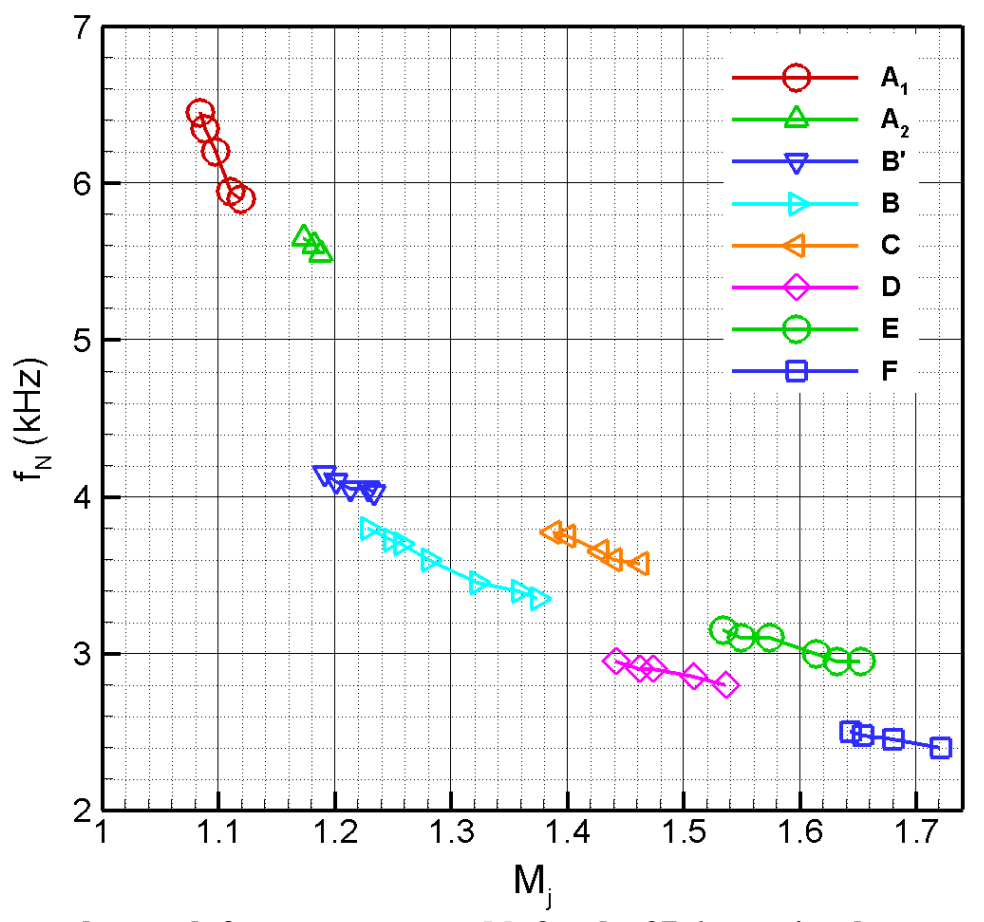

Fig. 9 Fundamental screech frequency versus $M_{j}$ for the $37.6 \mathrm{~mm}$ circular convergent nozzle. 

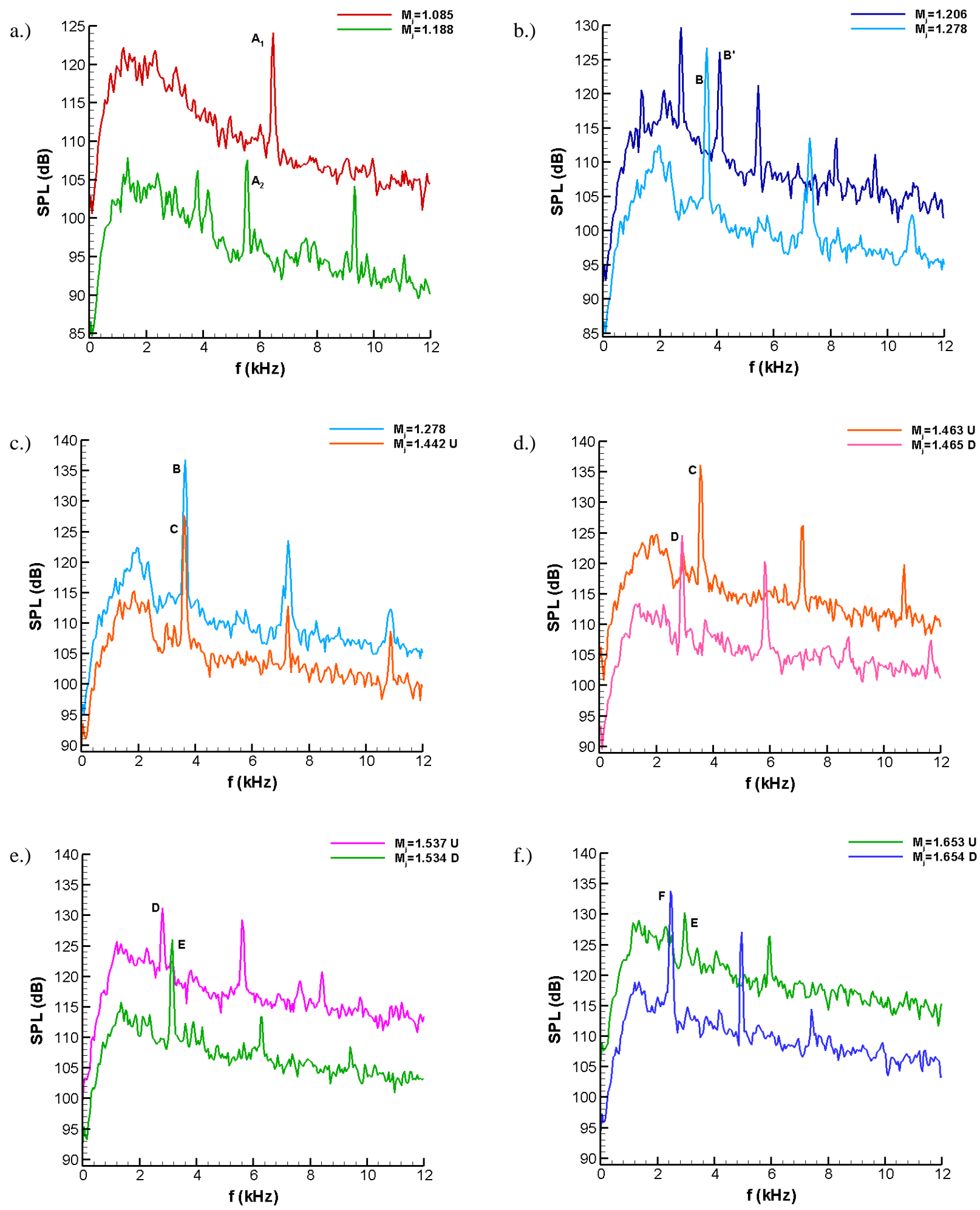

Fig. 10 Sound pressure level spectra for various screech stages. (a) Spectra from the non-overlapping stages $A_{1}$ and $A_{2}$, (b) stages B' and B, (c) B and C, (d) C and D, (e) D and E and (f) E and F. Suffix ' $U$ ' (or no suffix) in the legend denotes the spectra were recorded for increasing $M_{j}$, whereas the letter ' $D$ ' denotes the spectra were recorded for decreasing $M_{j}$.

8

American Institute of Aeronautics and Astronautics 

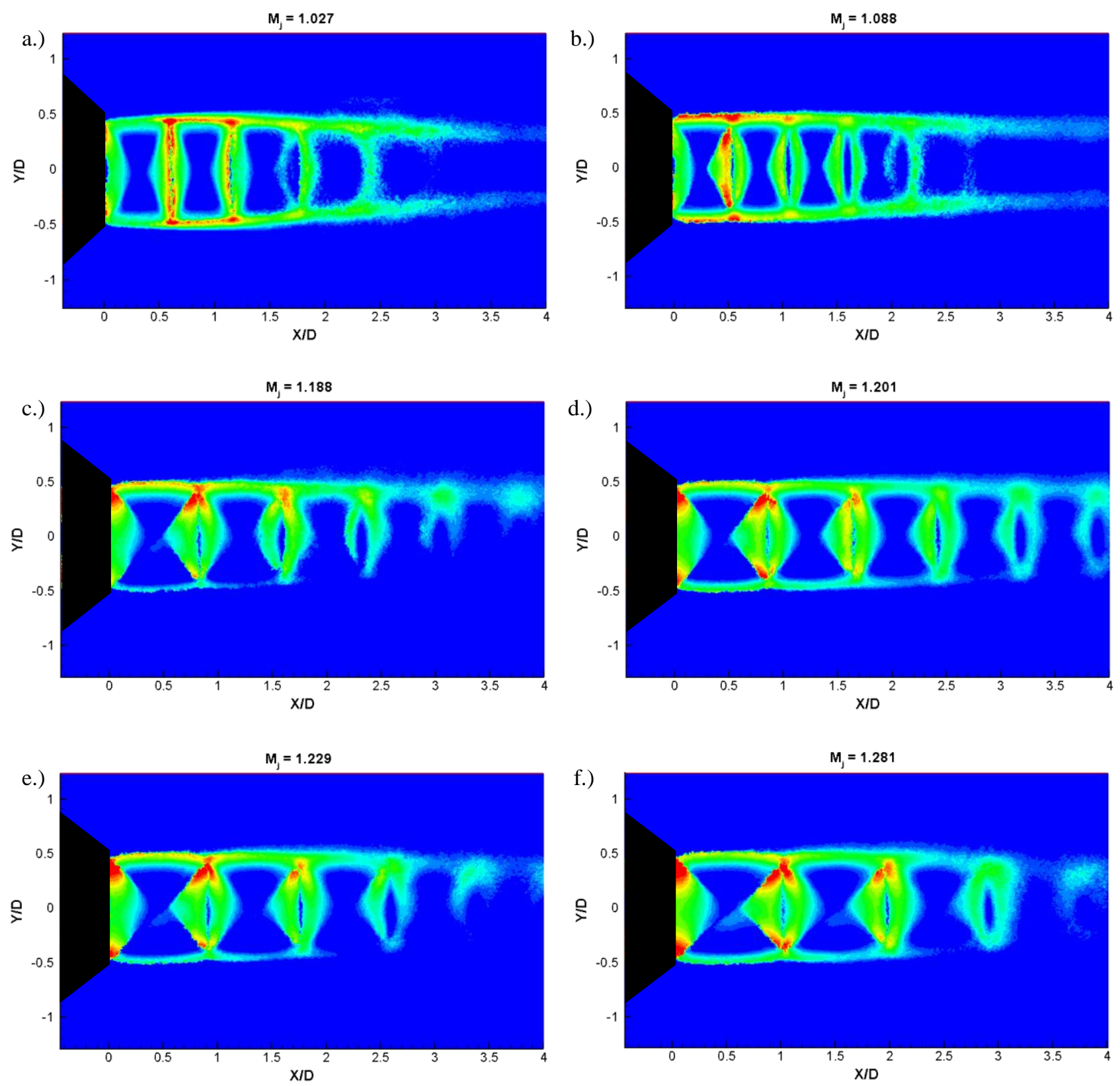

Fig. 11 BOS images at different $M_{j}$, (a) without any perceptible screech, (b) stage $A_{1}$, (c) stage $A_{2}$, (d) stage B', (e) stages B'+B, (f) stage B. (Figure continued on next page) 

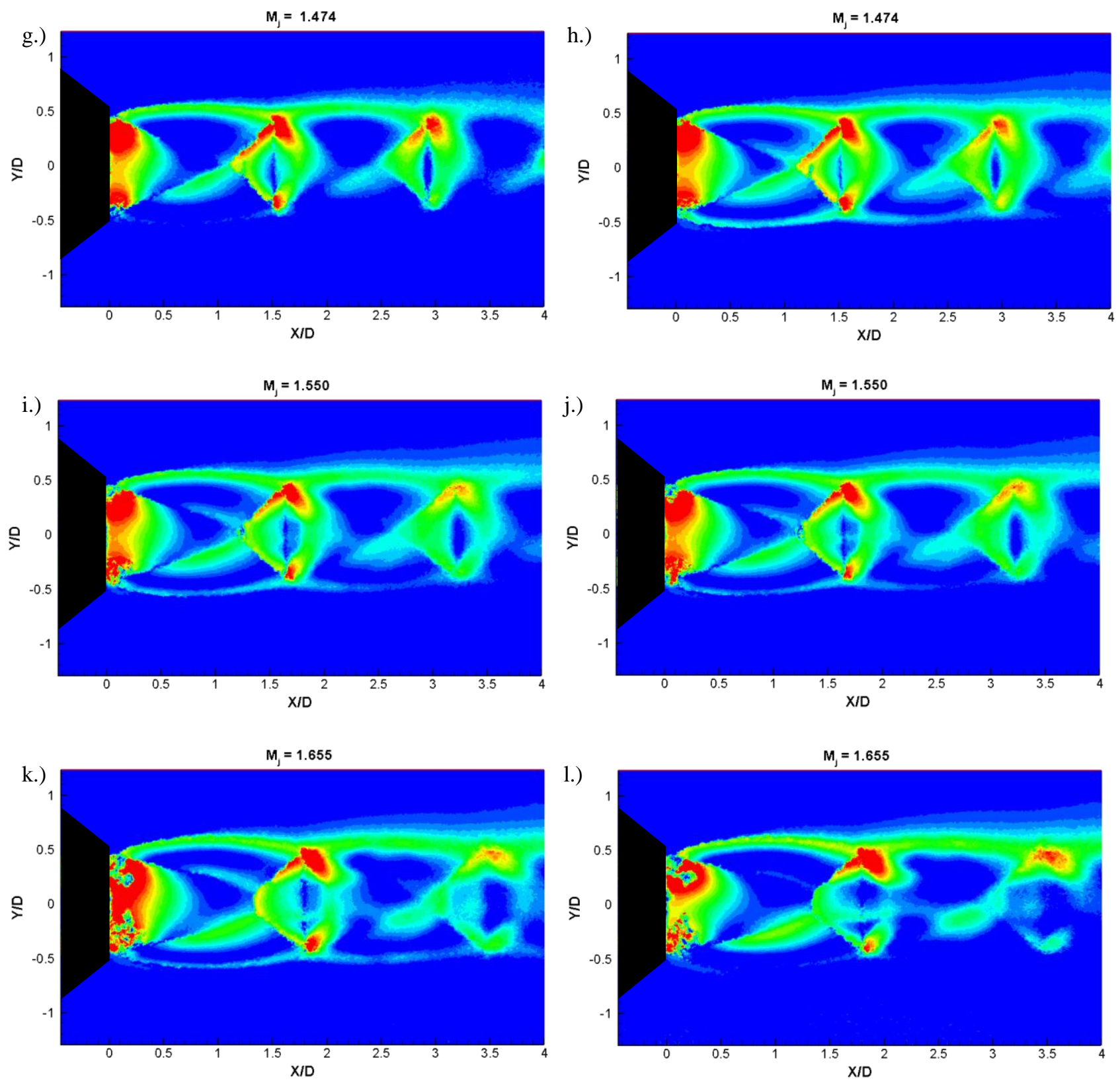

Figs. 11 BOS images at different $M_{j}$, (g,h) C and D stages within hysteresis loop for increasing $M_{j}$ and then decreasing $M_{j}$, respectively, $(\mathrm{i}, \mathrm{j}), \mathrm{D}$ and $\mathrm{E}$ stages within hysteresis loop, $(\mathrm{k}, \mathrm{l}) \mathrm{E}$ and $\mathrm{F}$ stages within hysteresis loop. 
From the BOS images, the average shock spacing was determined in the manner previously described in section III. A. The resulting non-dimensional shock spacing measured as a function of $M_{j}$ is shown in Fig. 12. It is apparent that the shock spacing follows a monotonic trend. As $M_{j}$ increases, the shock spacing increases as well. ${ }^{6}$ Large departures from this trend, beyond the uncertainty of the data, do not appear over the entire range of $M_{j}$ covered in the experiment. At the beginning of the experiment, it was suspected that the shock spacing would display an abrupt change for different stages at the $M_{j}$ values where the hysteresis occurred. No such change did occur. Therefore, it is inferred that the shock spacing is not the parameter that adjusts to accommodate a new frequency when a stage jump occurs. Other parameters, such as convection velocities in different segments of the feedback loop or the distance over which the instability wave develops nonlinearity, possibly adjust to provide for the stage jumps.

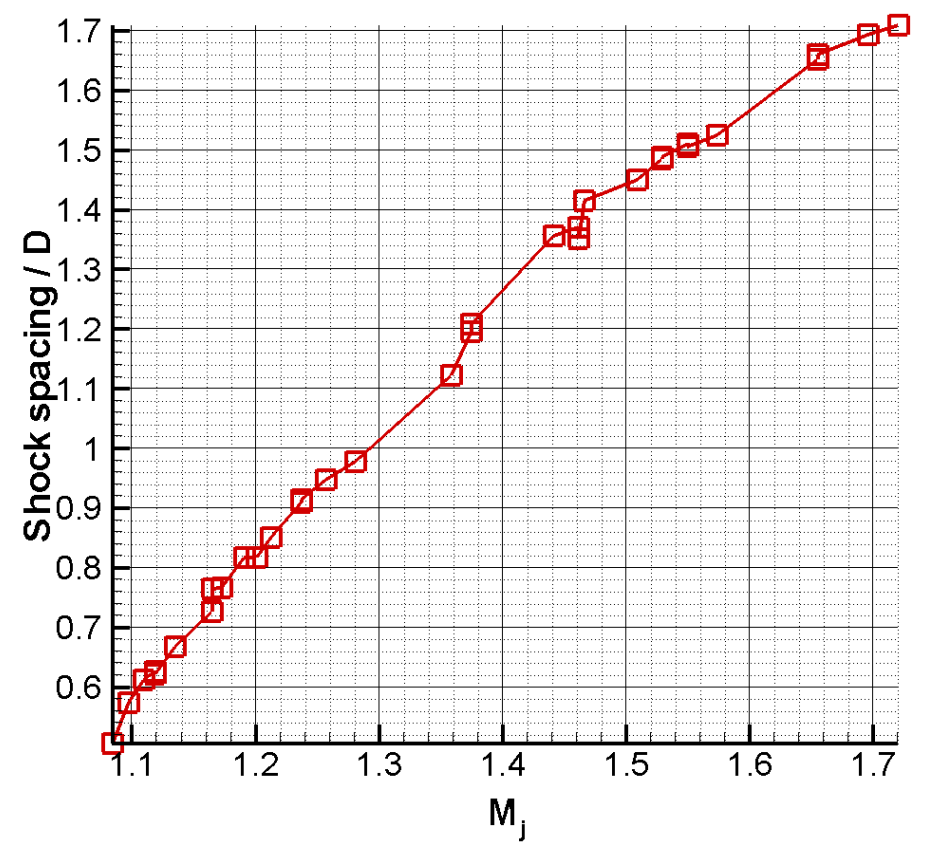

Fig. 12 Non-dimensional shock spacing versus $M_{j}$ corresponding to the frequency data shown in Fig. 9.

\section{Conclusions}

Background oriented schlieren (BOS) images were compared with shadowgraph data acquired under the same conditions. The average shock spacings obtained by the two techniques, in a convergent-divergent nozzle with design Mach number of 1.8, were shown to be consistent with one another. Although the resulting shadowgraph and BOS images had comparable spatial resolutions, there were differences in the images. The BOS images were not as crisp as the shadowgraph data and showed some asymmetry. This is thought to be due to lack of complete refinement of the BOS application in the present setup, e.g., background and the image processing. However, the shock spacing measured by BOS was found to be consistent with the shadowgraph data. This provided confidence in the shock spacing measurement by the BOS technique which was then used to investigate mode-switching behavior associated with the screech phenomenon. Screech frequencies from a circular convergent nozzle were collected over a jet Mach number range of $1.0<M_{j}<1.7$. The data exhibited as many as eight 'stages' within the $M_{j}$ range covered. BOS images were acquired at different screech conditions with special attention to regions of stage jumps. The shock spacings from the BOS images were shown to follow a monotonic trend with varying $M_{j}$ without any discontinuities across the stage jumps. Thus it was concluded that the stage jumps did not occur due to an adjustment of shock spacing. Other parameters, such as convection velocities in different segments of the feedback loop or the distance over which the instability wave develops nonlinearity, possibly adjust to account for the stage jumps. 


\section{References}

${ }^{1}$ Venkatakrishnan, L., and Meier, G. E. A, "Density measurements using the Background Oriented Schlieren Technique," Experiments in Fluids, Vol. 37, 2004, pp., 237-247.

${ }^{2}$ Hargather, M. J., Settles, G. S., "Recent Developments in Schlieren and Shadowgraph,” AIAA Paper 2010-4206, 2010.

${ }^{3}$ Richard, H., and Raffle, M., "Principle and applications of the background oriented schlieren (BOS) method," Measurement Science and Technology, Vol. 12, 2001, pp., 1576-1585.

${ }^{4}$ Tam, C. K. W., "Supersonic Jet Noise," Annual Rev. Fluid Mech., Vol. 27, 1995, pp., 17-43.

${ }^{5}$ Powell, A., "On the Mechanism of Choked Jet Noise," Proc. Phys. Soc. London, Vol. B 66, 1953, pp., 1039-1056.

${ }^{6}$ Raman, G., "Cessation of Screech in Underexpanded Jets," J. Fluid. Mech., Vol. 336, 1997, pp., 69-90.

${ }^{7}$ Norum, T. D., "Screech Suppression in Supersonic Jets," AIAA J., Vol. 21, 1983, pp., 235-240.

${ }^{8}$ Zaman, K. B. M. Q., "Spreading Characteristics of Compressible Jets from Nozzles of Various Geometries," J. Fluid. Mech., Vol. 383, 1999, pp., 197-228.

${ }^{9}$ Norum, T. D., "Control of Jet Shock Associated Noise by a Reflector," AIAA Paper 84-2279, 1984.

${ }^{10}$ Zaman, K. B. M. Q., Bencic, T. J., Clem, M. M., and Fagan, A. F., "Shock-induced boundary layer separation in C-D nozzles and its impact on jet noise," AIAA Paper 2011-1031, 2011. 